\title{
Lipocalin gene expression is varied in developmental stages by larval nutritional stress in Drosophila
}

\author{
Nazlı AYHAN ${ }^{1,2}$, Pınar GÜLER ${ }^{1,3}$, Banu Şebnem ÖNDER ${ }^{1, *}$ \\ ${ }^{1}$ Department of Biology, Genetics Section, Faculty of Science, Hacettepe University, Beytepe, Ankara, Turkey \\ ${ }^{2}$ UMR_D 190 “Emergence des Pathologies Virales”, Aix Marseille University, French Institute of Research for Development (IRD), \\ French School of Public Health (EHESP), Marseille, France \\ ${ }^{3}$ Institute for Zoology, University of Regensburg, Regensburg, Germany
}

\author{
Received: 08.04.2016
}

- $\quad$ Accepted/Published Online: 22.09.2016

- $\quad$ Final Version: 20.02 .2017

\begin{abstract}
Lipocalin genes NLaz, GLaz, and Karl are evolutionarily conserved genes in Drosophila melanogaster. There are studies on lipocalin gene expression differences under diverse diet conditions, but these studies have focused mainly on age-dependent expression profiles of these genes. The main aim of our study is to determine lipocalin expression in the developmental period by nutritional manipulation with an isofemale-based design. Three larval developmental periods have been researched under normal and restricted diets. We found significant differences between lines during their developmental time-related lipocalin expression. Here, we demonstrate that upregulations in the early developmental stages of lipocalin genes under stressful conditions resulted in unaffected developmental time. The possible reason for high expression is the activation of stress signal pathways in order to buffer the harmful effects of nutritional restriction. Our data showed that the early developmental period $(48-72 \mathrm{~h})$ is especially crucial to tolerate the dietary stress with respect to GLaz and NLaz expression. Results of this experiment have shown that the expression profiles of lipocalin genes have line-specific pathways to nutritional stress. Their expression depends on the genetic background corresponding to development time results. Our results highlight the transcriptional changes of lipocalins associated with developmental time in larvae, developed in a dietary-restricted medium.
\end{abstract}

Key words: Dietary stress, lipocalin gene family, ApoD, developmental time, Drosophila melanogaster

\section{Introduction}

Maintaining metabolic homeostasis is crucial for multicellular organisms (Torday and Rehan, 2012); in particular, developmental stage stability under changing environmental conditions has an important role for organisms' adaptation to the environment (Flatt and Heyland, 2011). When organisms encounter environmental stress, the characters of organisms are shaped by evolutionary adaptive mechanisms to buffer the environmental stress factors (Koehn and Bayne, 1989; Bijlsma and Loeschcke, 2005).

In nature, one of the most common environmental stresses is nutritional stress (Robbins, 1983). To imitate nature, dietary restriction has been used artificially in life history studies for almost a century. The first record of correlation between lifespan and nutrition was shown in white rats by McCay et al. (1935). Studies that followed focused on the dietary effects on different life history traits such as developmental time, lifespan, fecundity, and body size in different model organisms (Robertson, 1960;

*Correspondence: bdalgic@hacettepe.edu.tr

178
Chippindale et al., 1993, 1997; Roth et al., 1999; Jiang et al., 2000; Lin et al., 2002; Shingleton et al., 2008; Metaxakis and Partridge, 2013; Zhu et al., 2014). These studies showed how organisms shape their life history traits under stressful conditions to buffer the harmful effects of unfavorable conditions by the regulation of stress signals (Chippindale et al., 1997; Minois, 2000; Tu and Tatar, 2003; Partridge et al., 2005; Burger et al., 2007; Doroszuk et al., 2012).

It is known that nutrition-dependent insulin signals have an important role in stress pathways (Schenk et al., 2008). One of the stress-responsive signaling pathways, c-Jun-N-terminal kinase (JNK), also known as the stressactivated protein kinase, has been identified in flies and worms (Giannakou et al., 2007; Wolf et al., 2008). Additionally, studies on lipocalin family genes showed that stress-responsive signal pathways have important roles in the regulation of lipocalin genes and metabolic adaptation of the organism to environmental challenges (Hull-Thompson et al., 2009; Pasco and Léopold, 2012; 
Ruiz et al., 2012). Lipocalin genes have diverse functions, which are protein transportation, cryptic coloration, olfaction, pheromone transport, the enzymatic synthesis of prostaglandins, and the mediation of cell homoeostasis (Flower, 1996). The lipocalin gene family is evolutionarily conserved from flies to vertebrates. Neural Lazarillo (NLaz) and glial Lazarillo (GLaz), which are expressed in the Drosophila brain during the development of nervous system, are two homolog genes of human lipocalin gene apo-lipoprotein D (ApoD) (Ruiz et al., 2011). The other lipocalin gene, Karl, is not modified in Drosophila's nervous system, unlike NLaz and GLaz (Hull-Thompson et al., 2009; Dassati et al., 2014).

Lipocalin family gene expression profile differences have been identified in various nutritional stress conditions. NLaz transcription's relationship with oxidative stress and JNK signaling in the fat body was reported in previous studies (Thompson, 2008; Hull-Thompson et al., 2009). Recent research on Drosophila with high sugar diets showed that NLaz and GLaz expression is affected by a high sugar diet, but Karl expression is not modified with differing nutrition (Pasco and Léopold, 2012). Additionally, high expression of NLaz inhibits growth while increasing the organism's stress and starvation resistance (HullThompson et al., 2009). For GLaz, the importance of gene expression for stress tolerance and lifespan was shown in former studies (Sanchez et al., 2006; Walker et al., 2006).

For holometabolous organisms, the developmental stage is a critical period for the nervous system due to environmental pressure (Rice and Barone, 2000). Developmental stage stability is highly related to organism fitness, which depends critically on growth and development. Developmental time has many different responses on other life history characters, such as short development time, decreasing sexual maturity age, and generation time, thus increasing preadult survival by shortening the developmental period, which has a high predatory risk. On the other hand, long developmental time leads to a longer growth period and bigger body size, which increases mating success (Stearns, 1992; Stearns et al., 2000). A negative correlation between dietary protein content and developmental time has been shown (Shingleton et al., 2008; Kolss et al., 2009). Restricted protein has an important role in larvae to pupae developmental time (Güler et al., 2014) due to different stress signals.

From this point of view, the main aim of our research was to identify gene expressions of NLaz, GLaz, and Karl during developmental time under standard and restricted food conditions. For that purpose, we designed an inbred line-based experiment to introduce genetic background effects to gene expression profiles. We compared developmental stage-related gene expressions between the larvae of long- and short-developed inbred lines with standard and restricted diets. We investigated the relationship between lipocalin genes and larvae development under unfavorable dietary conditions for both lines. This study provides the first description of dietary effects on lipocalin transcriptional phenotypes in D. melanogaster larval development. Furthermore, our results identify the importance of lipocalin genes acting as a part of a stress response that adjusts developmental time in response to dietary restriction.

\section{Materials and methods}

\subsection{Drosophila strains and maintenance}

D. melanogaster populations were collected from Firtına Valley, Turkey, in 2009. One hundred isofemale lines were generated from females and all lines were maintained in standard laboratory conditions $\left(25^{\circ} \mathrm{C}, 12\right.$-h light and 12 -h dark cycle, $55 \pm 5 \%$ humidity). Fruit flies were reared in a standard sugar/yeast/agar (SY) medium ( $50 \mathrm{~g} / \mathrm{L}$ yeast, $50 \mathrm{~g} / \mathrm{L}$ sugar, $10 \mathrm{~g} / \mathrm{L}$ agar, and $30 \mathrm{ml} / \mathrm{L}$ Nipagin based on the protocol of Bass et al. (2007)). All handling of flies was performed at room temperature by using $\mathrm{CO}_{2}$ anesthesia. For experiments, we used a standard diet and a restricted diet, which were modified only by dilution of yeast $(10$ $\mathrm{g} / \mathrm{L})$.

We used an isofemale-based design, which is one of the most common methods in population genetics studies with Drosophila (David et al., 2005).

\subsection{Developmental time measurement techniques}

Ten isofemale lines were randomly chosen among stock colonies. For the developmental time experiment, adults were maintained in small chambers with agar and yeast paste petri dishes. After $24 \mathrm{~h}$ of incubation time, first-instar larvae were collected from each line. Each vial included 20 first-instar larvae and 10 vials (technical replicates) were used for the standard diet and 15 replicates for the restricted diet. The number of pupae and adults emerging from these cultures was recorded every $12 \mathrm{~h}$. Larva to pupa, pupa to adult, and larva to adult developmental times were recorded for each line for the two different diet regimes.

Two lines that showed high and low developmental time differences under standard and restricted diets were selected based on their developmental time data.

After the selection, the developmental time experiment was repeated for the selected lines. First-instar larvae were collected from standard and restricted diet groups by using small chambers as described previously. At 48,72 , and 96 h, 5-10 larvae were picked up from the food medium and stored at $-80^{\circ} \mathrm{C}$ for molecular studies.

2.3. RNA isolation, cDNA synthesis, and real-time qPCR RNA was isolated from 5-10 pooled larvae by homogenization in QIAzol Lysis Reagent (QIAGEN) 
and treated with DNase. The RNA was quantified using a NanoDrop 2200 (Thermo Fischer Scientific). Total RNA was converted into cDNA using the Thermo Scientific RevertAid First Strand cDNA Synthesis kit with oligo (dT) primers. For all lines, we performed two biological and two technical replicates. Real-time PCR wasperformed using the QuantiTect SYBR Green PCR Kit (QIAGEN) in a QIAGEN Rotor Gene machine with a mixture of primers according to the manufacturer's instructions. Expression of each gene relative to the housekeeping genes $\alpha$-tubulin and eIF$1 A$ was determined by the $\Delta \Delta \mathrm{Ct}$ method. The following PCR primers were used: NLaz primers, forward: 5'-CATTGACGAAGGCCAACTA-3', reverse 5'-GGATACATCGTTGTCTTCCA-3'; GLaz primers, forward: $5^{\prime}$-AGATGCCTACGGATTTGGA-3', reverse 5'-TACGGCTCAAACTGAAAGG-3'; Karl primers, forward: 5'-GGTTCAGTATTACGCATCCAC-3', reverse 5' ${ }^{\prime}$-TATATGTCCTCCGTGTGCTG- ${ }^{\prime} ; \alpha$-tubulin primers, forward: $\quad 5^{\prime}$-GTTTGTCAAGCCTCATAGCC-3', reverse $5^{\prime}$-TGGATAGAGATACATTCACGCA-3'; eIF-1A primers, forward: 5'-TCACATTCGGGGGAAACTTCG-3', reverse 5'-GTACGTCTTCAGGTTCCTGGC-3'. Cycling conditions were $15 \mathrm{~min}$ at $95^{\circ} \mathrm{C}\left(15 \mathrm{~s} 94^{\circ} \mathrm{C}, 30 \mathrm{~s} 55^{\circ} \mathrm{C}, 30\right.$ $\left.\mathrm{s} 72^{\circ} \mathrm{C}\right) \times 45$, followed by $5 \mathrm{~min}$ at $90^{\circ} \mathrm{C}$. Gene regions of $146 \mathrm{bp}, 120 \mathrm{bp}$, and $169 \mathrm{bp}$ were amplified respectively for NLaz, GLaz, and Karl.

\subsection{Analysis}

Nested analyses of variance (ANOVAs) were used to test the effects of protein concentration on larva to pupa and larva to adult developmental time. One-way ANOVA was used to test the statistical significance of the differences between larval development on standard and restricted food. All statistical analyses were done using SPSS 20.
Gene expression analyses were done with SA Bioscience RT2 Profiler PCR Array Data Analysis Version 3.5 (http:// pcrdataanalysis.sabiosciences.com/pcr/arrayanalysis. $\mathrm{php}$ ). This software calculated fold regulation values for each gene using the relative quantification $2^{-\Delta \Delta \mathrm{Ct}}$ method (Livak and Schmittgen, 2001). $\Delta \mathrm{Ct}$ values were normalized using the mean values of two housekeeping genes: $\alpha$-tubulin and eIF-1A. Student's t-test was performed with obtained data using SPSS 20.

\section{Results}

Based upon the knowledge of environmental stress signal pathways' effects on lipocalin genes expression profiles, we investigated the gene expression profiles of GLaz, NLaz, and Karl under standard and restricted protein conditions during developmental stages. We chose "developmental time" as a critical parameter because it is known to be affected by nutritional conditions. We focused on the expression changes in the larvae stages and investigated how much of their expression is contributed by the developmental stages. These data can explain the hypothetical relationship between developmental time and lipocalin expression, which is known to regulate stress resistance in fruit flies. To select the isofemale lines for gene expression studies, preexperiments were performed to investigate developmental time. We found variable effects of developmental time by diet restriction. The results of the two-way nested ANOVA of developmental time are presented in the Table. The line and diet effects on variation of developmental time were highly significant in both developmental stages $(\mathrm{P}<0.001)$. Genetic changes often have quantitatively variable effects on an organism's phenotype in different genetic backgrounds and in different environments. Figure 1 represents developmental

Table. Two-way nested ANOVAs testing for differences in mean developmental time between isofemale lines, diets, and their interactions.

\begin{tabular}{|l|l|l|l|l|}
\hline Source of variation & df & Mean square & F & P \\
\hline (a) Larva to pupa developmental time & \multicolumn{5}{l|}{} \\
\hline Isofemale line & 9 & $12,011.843$ & 38.889 & $<0.001$ \\
\hline Diet & 1 & $213,991.971$ & 692.819 & $<0.001$ \\
\hline Isofemale line $\times$ diet & 9 & 2515.197 & 8.143 & $<0.001$ \\
\hline Error & 2694 & 308.872 & & \\
\hline (b) Larva to adult developmental time & \multicolumn{5}{|l|}{} \\
\hline Isofemale line & 9 & $13,500.994$ & 34.420 & $<0.001$ \\
\hline Diet & 1 & $389,051.271$ & 991.861 & $<0.001$ \\
\hline Isofemale line $\times$ diet & 9 & 5385.242 & 13.729 & $<0.001$ \\
\hline Error & 9 & 392.244 & & \\
\hline
\end{tabular}




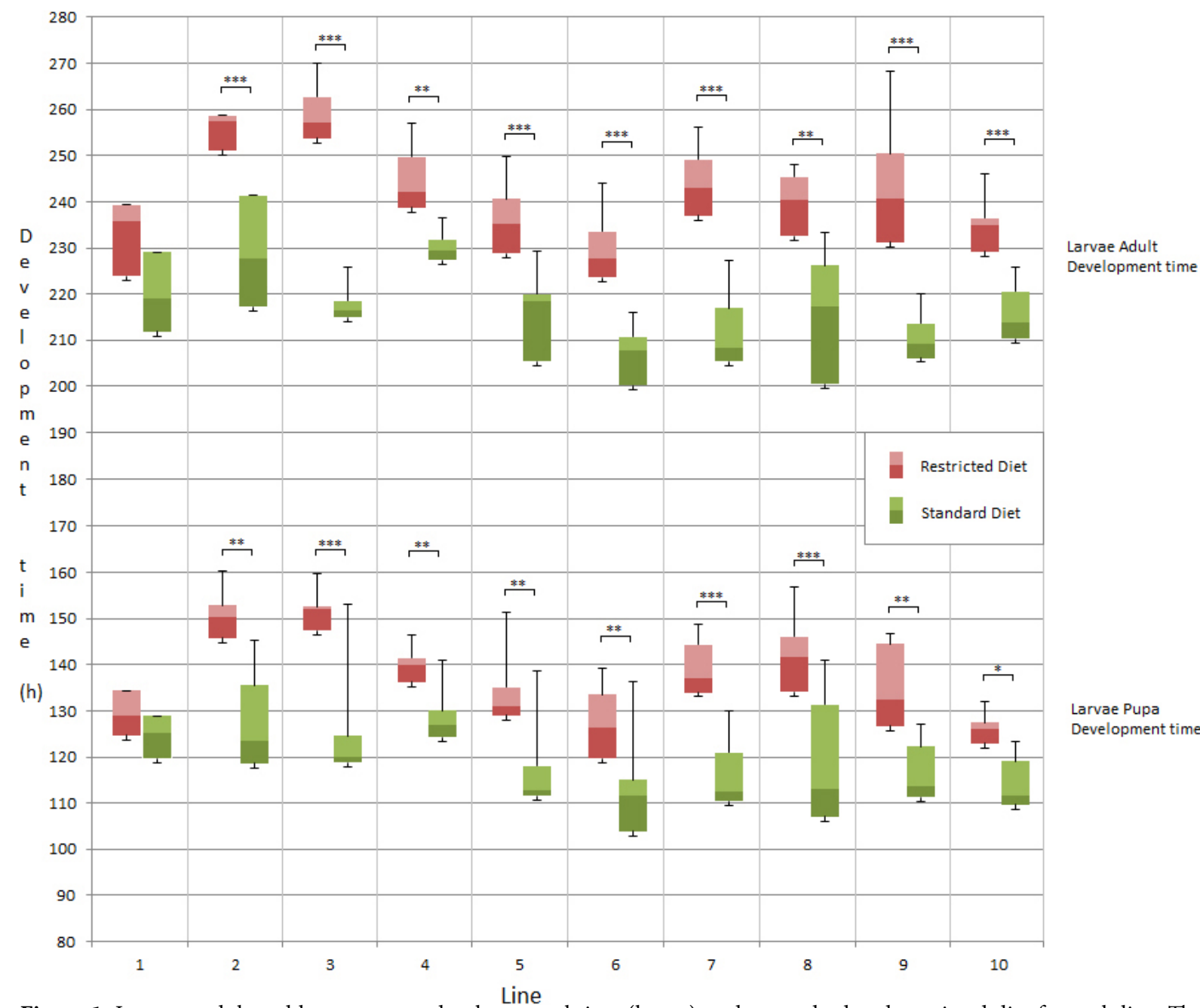

Figure 1. Larva to adult and larva to pupa developmental time (hours) under standard and restricted diet for each line. The $\mathrm{y}$-axis shows the developmental time in hours. The $\mathrm{x}$-axis shows the isofemale lines coded from 1 to 10 , and error bars show standard errors of the means. One-way ANOVA with Bonferroni post hoc test was used for comparisons. Significant levels are indicated: ${ }^{\star} \mathrm{P}<0.05 ;{ }^{* *} \mathrm{P}<0.01 ;{ }^{* *} \mathrm{P}<0.001$.

time from larva to pupa and larva to adult under two different diet conditions for ten isolines. Developmental time data showed genotype-dependent responses. Oneway ANOVA with a Bonferroni post hoc comparison was performed to determine the effect of diet.

Although each line had a specific pathway for its developmental time, almost all lines except the first line showed statistically longer developmental time under nutritional stress, especially from the larva to pupa period (Figure 1). This result is confirmed by previous research and literature (Onder and Yilmaz, 2009; Önder, 2010; Matzkin et al., 2011; Güler et al., 2014; Neethu et al., 2014); a negative relationship has been shown between the reachability of a protein resource and developmental time.

For gene expression studies we chose two lines out of ten to investigate developmental time in standard and restricted dietary conditions. One of the chosen lines was the first line, which showed no statistically significant developmental time difference between the larvae raised on standard versus restricted diets (Figure 1). The second was the 7th line, which showed significant developmental time differences between standard and restricted diets, unlike Line 1 (Figure 1).

To further investigate the developmental time differences between Line 1 and Line 7, we focused on agerelated expression in different stages of the development period. Figure 2 presents NLaz, GLaz, and Karl expressions under standard and restricted diet conditions in three different periods of the developmental stages: 48,72 , and $96 \mathrm{~h}$ of larval development. The relative contribution of different developmental stages in lipocalin expressions also varied with lines. Additionally, our analyses strongly 
Line 1

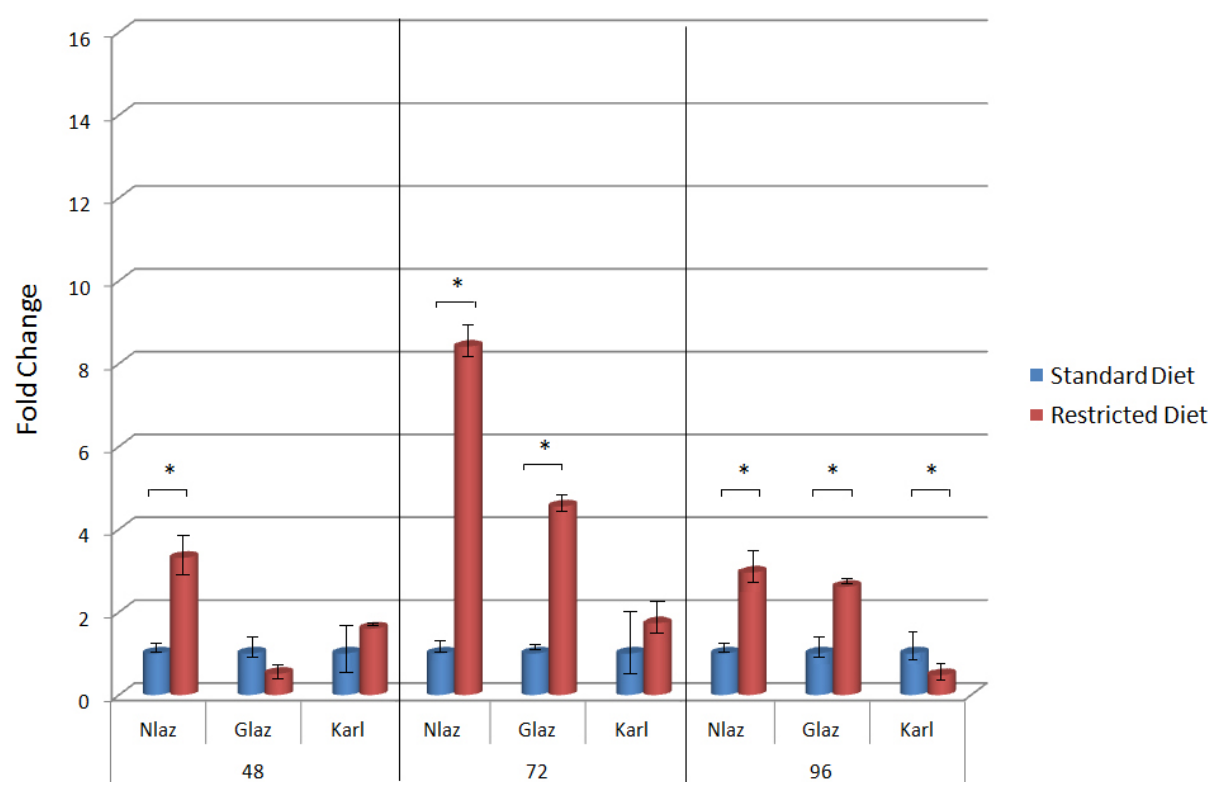

Line 7

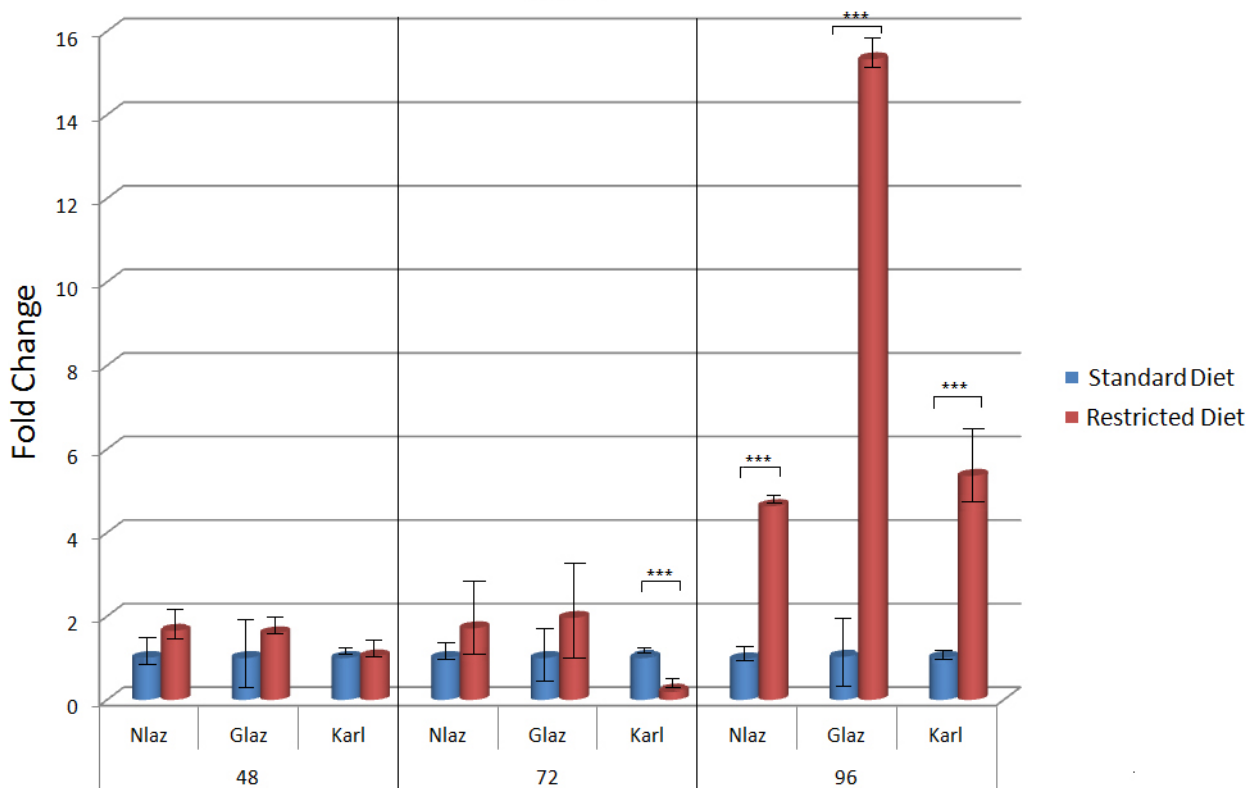

Figure 2. Change of NLaz, GLaz, and Karl gene expressions under standard and restricted diet conditions for two selected lines: Line 1 and Line 7 . The y-axis shows the fold change of expression levels (mean $\pm \mathrm{SE}$ ). The $\mathrm{x}$-axis shows the genes for each analyzed developmental time period in hours. Standard diet conditions were used as the control. Error bars show standard errors of the means. Significance was determined by Student's t-test. ${ }^{* *} \mathrm{P}<0.01{ }^{* * *} \mathrm{P}<0.001$.

suggest that the difference in growing larvae on restricted diet between Line 1 and Line 7 is associated with changes in the expression of lipocalin genes. Diet affected gene expression for the lines we analyzed. Larvae of Line 1 had stable Karl expression levels with a slight decrease at $96 \mathrm{~h}$ in all investigated developmental stages. Among the other tested molecules in Line 1 we found that transcription of the lipocalin NLaz was potently induced in 72-h-old larvae grown on restricted medium. Larvae raised on restricted diets showed an eightfold increase in NLaz expression as measured by RT-qPCR (Figure 2). Similarly, GLaz expression showed a fourfold increase in the same 
experimental group. Additionally, at $96 \mathrm{~h}$ larvae raised on restricted diets showed a twofold increase in NLaz and GLaz expression in Line 1. However, in Line 7, NLaz and GLaz were only significantly upregulated at $96 \mathrm{~h}$ in larvae raised on the restricted diet. GLaz showed a 15 -fold increase at this developmental time point (Figure 2).

Interestingly, the levels of lipocalins were correlated with the developmental time of the selected lines. When lipocalin expression in restricted medium did not differ from the standard medium in the 48-h and 72-h larvae, developmental time was affected in an extended way. Otherwise, if lipocalin expressions increased in early developmental stages, developmental time was minimally affected by dietary stress.

\section{Discussion}

The main objective of our study was to identify the lipocalin gene expression profiles in larval development under two different diet conditions. We used two isofemale inbred lines selected according to their developmental time on standard and restricted diets. The selected lines' larvae were collected at three different development points, 48,72 , and $96 \mathrm{~h}$, and analyzed for lipocalin gene expressions for the two diets. The results of the lines' developmental time and lipocalin gene expression contribute to the understanding of the relationships between diet stresses, lipocalin genes, and developmental time. This is an important result of this study because most previous studies mainly focused on lipocalin gene expression changes in adult flies.

We found that the gene expression of NLaz for Line 1 increased significantly under stressful conditions for each developmental stage. Line 7, which showed significantly high developmental time differences between restricted and standard diet conditions, also showed similar profiles for NLaz gene expression at 48 and $72 \mathrm{~h}$ under standard (control) and restricted conditions. Flies lacking in NLaz have decreased energy storage and show a decreased resistance to starvation; however, overexpression of $\mathrm{NLaz}$ results in an increase of starvation resistance (Rong et al., 2002). In agreement, Hull-Thompson et al. (2009) showed that loss of NLaz function reduces stress resistance and lifespan in flies. Accordingly, NLaz is transcriptionally regulated by JNK signaling and is required for JNKmediated stress and starvation tolerance. Reduced activity of the insulin/insulin-like growth factor signaling (IIS) pathway increased stress tolerance and prolonged lifespan (Kapahi et al., 2004), consistent with phenotypes occurring in the overexpression of NLaz (Hull-Thompson et al., 2009). We demonstrated that expression of the NLaz gene was higher in restricted groups than standard in all analyzed developmental time groups and that it increased with the developmental period in Line 1 while showing a sharp increase at $72 \mathrm{~h}$. Differently, in Line 7 NLaz remained at a similar level to that of the control at the beginning at the developmental period and showing a sharp increase at $96 \mathrm{~h}$. These results follow the previous findings from the phenotypical level, where higher expression levels of NLaz were found in relation to increased stress tolerance (Rong et al., 2002; Hull-Thompson et al., 2009). JNK signaling is activated in flies as a result of dietary restriction (Clancy et al., 2002). However, JNK signaling influences IIS signaling indirectly through the action of lipocalin signals (HullThompson et al., 2009). These findings showed that NLaz expressed in early developmental stages was characterized by the same developmental time in the two dietary conditions as compared with those in late-expressed developmental stages, with extended developmental time in a dietary-restricted medium. The comparisons of the developmental stage-dependent expression between the lines showed divergent expression patterns between the lines. We can speculate that increasing expression of the NLaz gene occurring in the early developmental period can be characterized by increased dietary stress tolerance in larval developmental time.

For GLaz expression in Line 1, an increase at 72 and 96 $\mathrm{h}$ but not at $48 \mathrm{~h}$ was detected as a probable reason for late development stage genetic expression. Line 1 is the line that showed no significant developmental time differences between restricted and standard diet conditions; in other words, dietary stress did not affect developmental time in Line 1. GLaz overexpression resulted in a longer lifespan and an increase in resistance to starvation and hyperoxia in adult flies (Walker et al., 2006). In contrast, GLaz-null mutant flies showed a decrease in mean lifespan and lower resistance to starvation (Sanchez et al., 2006). It is known that GLaz expression is increased with a high sugar diet (Pasco and Leopold, 2012) and stress like hyperoxia, high temperatures, and paraquat exposure (Muffat et al., 2008). From these points of view, it can be speculated that restricted yeast in the diet leads to a stress response at the GLaz expression level. The overexpression of GLaz and $N L a z$ together protects the larvae from the detrimental effects of diet restriction on developmental duration. In the analysis of Line 7, like NLaz, the expression of GLaz between standard (control) and restricted groups only differed significantly in the late stages of larval development at $96 \mathrm{~h}$. This analysis indicated that the developmental hour-related upregulation of the lipocalins under dietary stress conditions can be involved in the developmental length. As a result of upregulation of NLaz and GLaz, it is likely that individuals could buffer the effects of nutritional stress. This result is quite similar to the previous study of Thompson (2008), which indicated that NLaz acts downstream of JNK to promote starvation and stress and tolerance-maintained metabolic homeostasis. With respect to increasing gene expressions of NLaz and GLaz, organisms become more resistant to dietary stress. The 
relationship between the NLaz and GLaz genes and stress resistance has been indicated by previous studies (Sanchez et al., 2006; Walker et al., 2006; Thompson, 2008; HullThompson et al., 2009).

Unlike NLaz and GLaz, Karl gene expression showed no significant increase during development in Line 1; on the contrary, Karl was downregulated at $96 \mathrm{~h}$ of the development period in Line 1 . These results correspond with Pasco and Léopold's study (2012), which showed that the expression of Karl is not modified by changing the sugar component. Previous work indicated that a $\mathrm{P}$ element insertion in the Karl gene leads to the extension of fly development time to about $100 \mathrm{~h}$ (Mensch et al., 2008). Therefore, Karl is a candidate gene that is associated with developmental time but transcriptional data about the study are not available. In Line 7, Karl's expression decreased in the restricted group at $72 \mathrm{~h}$ of the development period. However, at $96 \mathrm{~h}$ high expression was shown under stressful conditions rather than the standard conditions for all lipocalin genes. A possible reason for the overexpression could be the pupation stage effects on metabolic homeostasis. Expression data show that $\mathrm{Karl}$ expression is very low in the developmental stages (Chintapalli et al., 2007); however, our results show that nutrient manipulation can lead to overexpression of Karl, dependent on the genetic background in developmental stages. An opinion is that the Karl gene modulates insulin signaling like NLaz (Hull-Thomson et al., 2009).

Our results have indicated major differences between Line 1 and Line 7 at the phenotypical and molecular levels. This is an interesting finding as the analyses performed for both lines and two diets indicate lipocalin genes as an important group in expression changes during the larval development in a distinct way.

High expression at late developmental time has no significant correlation with developmental time unaffected by dietary restriction. The late developmental period might be too late to tolerate nutritional stress effects for developmental time for the possible reason of the coming pupation stage. Our overexpression of lipocalins shortly before pupation may prolong the time taken to reach the critical size required for pupation. Despite this speculation, considering the relationship between stress tolerance and lipocalins in Drosophila, it can be suggested that early larval

\section{References}

Bass TM, Grandison RC, Wong R, Martinez P, Partridge L, Piper MDW (2007). Optimization of dietary restriction protocols in Drosophila. J Gerontol A Biol Sci Med Sci 62: 1071-1081.

Bijlsma R, Loeschcke V (2005). Environmental stress adaptation and evolution: an overview. J Evol Biol 18: 744-749. developmental periods are more important for genetic buffering to dietary stress. Generally, there is no specific expression pathway for time-dependent developmental periods, unlike age-specific expression. Despite Ruiz et al. (2011) showing age-specific NLaz and GLaz expression for each sex in D. melanogaster, it is hard to identify developmental stage-specific expression. On the other hand, GLaz and NLaz show low levels of larval expression and are highly expressed in pupae and adult flies (Sanchez et al., 2000; Maynard, 2010). Likewise, Karl's expression is very low during developmental stages (Chintapalli et al., 2007). Contrarily, our results do not support these findings. The tested lines show lipocalin expression at different time points of larval developmental stages by dietary stress. Moreover, the study by Mensch et al. (2008) suggested that the mutated Karl gene with $\mathrm{P}$ element insertion as a candidate affects developmental time. Our results highlight the large potential of the relationship between lipocalin genes and developmental time.

It is possible that lipocalin expression was buffered in the optimum diet composition in comparison to the lipocalins expressed in the yeast-restricted poor diet. However, differences of lipocalin expressions in both lines indicated that the genetic background of inbred lines has strongly influenced the transcription phenotype.

The main goal of this study was to identify the expression profile of lipocalin genes in larval developmental periods under nutritional stress; it needs to be noted that the effects of developmental time can be related to lipocalin genes. We identified NLaz, GLaz, and Karl expressions during development under two different diet conditions. This framework needs to be investigated in more detail based on tissue and molecular pathways to understand the nutritional effects of lipocalin gene expressions during the developmental stage.

\section{Acknowledgments}

We would like to thank Yasemin Ekmekçi, Can Koşukcu, and Ekim Taşkıran for their valuable advice and technical support during the planning and carrying out of this work. We are also grateful to two anonymous reviewers for useful comments. This work was supported by the Scientific and Technological Research Council of Turkey (TÜBİTAK Grant No. 212T170).

Burger JMS, Hwangbo DS, Corby-Harris V, Promislow DEL (2007). The functional costs and benefits of dietary restriction in Drosophila. Ageing Cell 6: 63-71.

Chintapalli VR, Wang J, Dow JAT (2007). Using Fly Atlas to identify better Drosophila melanogaster models of human disease. Nat Genet 39: 715-720. 
Chippindale AK, Leroi AM, Kim SB, Rose MR (1993). Phenotypic plasticity and selection in Drosophila life-history evolution. I. Nutrition and cost of reproduction. J Evol Biol 6: 171-193.

Chippindale AK, Leroi AM, Saing H, Borash DJ, Rose MR (1997). Phenotypic plasticity and selection in Drosophila life history evolution. 2. Diet mates and the cost of reproduction. J Evol Biol 10: 269-293.

Clancy DJ, Gems D, Hafen E, Leevers SJ, Partridge L (2002). Dietary restriction in long-lived dwarf flies. Science 296: 319.

Dassati S, Waldner A, Schweigreiter R (2014). Apolipoprotein D takes center stage in the stress response of the aging and degenerative brain. Neurobiol Aging 35: 1632-1642.

David JR, Gibert P, Legout H, Petavy G, Capy P, Moreteau B (2005). Isofemale lines in Drosophila: an empirical approach to quantitative trait analysis in natural populations. Heredity 94 : 3-12.

Doroszuk A, Jonker M, Pul N, Breit T, Zwaan B (2012). Transcriptome analysis of a long-lived natural Drosophila variant: a prominent role of stress- and reproduction-genes in lifespan extension. BMC Genomics 13: 167.

Flatt T, Heyland A (2011). Mechanisms of Life History Evolution: The Genetics and Physiology of Life History Traits and TradeOffs. Oxford, UK: Oxford University Press.

Flower D (1996). The lipocalin protein family: structure and function. Biochem J 318: 1-14.

Giannakou ME, Goss M, Jacobson J, Vinti G, Leevers SJ, Partridge L (2007). Dynamics of the action of dFOXO on adult mortality in Drosophila. Aging Cell 64: 29-38.

Güler P, Ayhan N, Koşukçu C, Önder BŞ (2014). The effects of larval diet restriction on developmental time pre-adult survival and wing length in Drosophila melanogaster. Turk J Zool 39: 395403 .

Hull-Thompson J, Muffat J, Walker DW, Benzer S (2009). Control of metabolic homeostasis by stress signaling is mediated by the lipocalin NLaz. PLoS Genet 5: e1000460.

Jiang JC, Jaruga E, Repnevskaya MV, Jazwinski SM (2000). An intervention resembling caloric restriction prolongs life span and retards aging in yeast. FASEB J 14: 2135-2137.

Kapahi P, Zid BM, Harper T, Koslover D, Sapin V, Benzer S (2004). Regulation of lifespan in Drosophila by modulation of genes in the TOR signaling pathway. Curr Biol 14: 885-890.

Koehn RK, Bayne RL (1989). Towards a physiological and genetical understanding of the energetics of the stress response. Biol J Linn Soc 37: 157-151.

Kolss M, Vijendravarma RK, Schwaller G, Kawecki TJ (2009). Lifehistory consequences of adaptation to larval nutritional stress in Drosophila. Evolution 63: 2389-2401.

Lin SJ, Kaeberlein M, Andalis AA, Sturtz LA, Defossez PA (2002). Calorie restriction extends Saccharomyces cerevisiae life span by increasing respiration. Nature 418: 344-348.

Livak KJ, Schmittgen TD (2001). Analysis of relative gene expression data using real-time quantitative PCR and the $2^{-\Delta \Delta C}$ method. Methods 25: 402-408.
Matzkin LM, Johnson S, Paight C, Bozinovic G, Markow TA (2011). Dietary protein and sugar differentially affect development and metabolic pools in ecologically diverse Drosophila. J Nutr 141: 1127-1133.

Maynard JC, Pham T, Zheng T, Jockheck-Clark A, Rankin HB, Newgard CB, Spana EP, Nicchitta CV (2010). Gp93, the Drosophila GRP94 ortholog, is required for gut epithelial homeostasis and nutrient assimilation-coupled growth control. Dev Biol 339: 295-306.

McCay CM, Crowell MF, Maynard LA (1935). The effect of retarded growth upon the length of lifespan and upon the ultimate body size. J Nutr 10: 63-79.

Mensch J, Lavagnino N, Carreira VP, Massaldi A, Hasson E, Fanara JJ (2008). Identifying candidate genes affecting developmental time in Drosophila melanogaster: pervasive pleiotropy and gene-by-environment interaction. BMC Dev Biol 8: 78.

Metaxakis A, Partridge L (2013). Dietary restriction extends lifespan in wild-derived populations of Drosophila melanogaster. PLoS ONE 8: e74681.

Minois N (2000). Longevity and ageing: beneficial effects of exposure to mild stress. Biogerontology 1: 15-29.

Muffat J, Walker DW, Benzer S (2008). Human ApoD an apolipoprotein up-regulated in neurodegenerative diseases extends lifespan and increases stress resistance in Drosophila. $\mathrm{P}$ Nat Acad Sci USA 105: 7088-7093.

Neethu BK, Ramesh BY, Harini BP (2014). Enhanced protein diet accelerates the rate of development. A study in Drosophila nasutanasuta. International Journal of Advanced Research 2: 349-354.

Önder BŞ (2010). A comparison of two different diets and their restricted groups in relation to developmental time and viability in Drosophila melanogaster. Hacettepe Journal of Biology \& Chemistry 38: 311-317.

Onder BS, Yilmaz M (2009). The effect of dietary restriction on developmental time in Drosophila melanogaster and its sibling D. simulans. Drosophila Information Service 92: 95-100.

Partridge L, Piper MDW, Mair W (2005). Dietary restriction in Drosophila. Mech Ageing Dev 126: 938-950.

Pasco MY, Léopold P (2012). High Sugar-Induced Insulin Resistance in Drosophila relies on the lipocalin Neural Lazarillo. PLoS ONE 7: e36583.

Rice D, Barone S (2000). Critical periods of vulnerability for the developing nervous system: evidence from humans and animal models. Environ Health Perspect 108: 511-533.

Robbins C (1983). Wildlife Feeding and Nutrition. New York, NY, USA: Academic Press.

Robertson FW (1960). The ecological genetics of growth in Drosophila: 1. Body size and developmental time on different diets. Genet Res 1: 288-304.

Rong YS, Titen SW, Xie HB, Golic MM, Bastiani M, Bandyopadhyay P, Olivera BM, Brodsky M, Rubin GM, Golic KG (2002). Targeted mutagenesis by homologous recombination in $D$. melanogaster. Genes Dev 16: 1568-1581. 
Roth GS, Ingram DK, Lane MA (1999). Calorie restriction in primates: will it work and how will we know? J Am Geriatr Soc 47: 896-903.

Ruiz M, Sanchez D, Canal I, Acebes I, Ganfornina MD (2011). Sex-dependent modulation of longevity by two Drosophila homologues of human apolipoprotein D GLaz and NLaz. Exp Geront 46: 579-589.

Ruiz M, Wicker-Thomas C, Sanchez D, Ganfornina MD (2012). Grasshopper Lazarillo, a GPI-anchored lipocalin, increases Drosophila longevity and stress resistance and functionally replaces its secreted homolog NLaz. Insect Biochem Mol Biol 42: 776-789.

Sanchez D, Ganfornina MD, Torres-Schumann S, Speese SD, Lora JM, Bastiani MJ (2000). Characterization of two novel lipocalins expressed in the Drosophila embryonic nervous system. Int J Dev Biol 44: 349-359.

Sanchez D, Lopez-Arias B, Torroja L, Canal I, Wang X, Bastiani MJ, Ganfornina MD (2006). Loss of glial lazarillo a homolog of apolipoprotein D reduces lifespan and stress resistance in Drosophila. Curr Biol 16: 680-686.

Schenk S, Saberi M, Olefsky JM (2008). Insulin sensitivity: modulation by nutrients and inflammation. J Clin Invest 118: 2992-3002.

Shingleton AW, Christen KM, Peter WB (2008). Developmental model of static allometry in holometabolous insects. P Roy Soc Lond B Bio 1645: 1875-1885.
Stearns SC (1992). The Evolution of Life Histories. Oxford, UK: Oxford University Press.

Stearns SC, Ackermann M, Doebeli M, Kaiser M (2000). Experimental evolution of aging growth and reproduction in fruit flies. P Natl Acad Sci USA 7: 3309-3313.

Thompson J (2008). Endocrine control of stress and metabolic signaling in Drosophila melanogaster. PhD, University of Rochester, Rochester, NY, USA.

Torday JS, Rehan VR (2012). Evolutionary Biology: Cell-Cell Communication and Complex Disease. Hoboken, NJ, USA: Wiley-Blackwell.

Tu MP, Tatar M (2003). Juvenile diet restriction and the aging and reproduction of adult Drosophila melanogaster. Aging Cell 2: 327-333.

Walker DW, Muffat J, Rundel C, Benzer S (2006). Overexpression of a Drosophila homolog of apolipoprotein D leads to increased stress resistance and extended lifespan. Curr Biol 16: 674-679.

Wolf M, Nunes F, Henkel A, Heinick A, Paul RJ (2008). The MAP kinase JNK-1 of Caenorhabditis elegans: location activation and influences over temperature-dependent insulin-like signaling stress responses and fitness. J Cell Physiol 214: 721-729.

Zhu CT, Ingelmo P, Rand DM (2014). G $\times \mathrm{G} \times \mathrm{E}$ for lifespan in Drosophila: mitochondrial nuclear and dietary interactions that modify longevity. PLoS Genet 10: e1004354. 